\title{
COMETARY ORIGIN OF CARBON AND WATER ON THE TERRESTRLAL PLANETS
}

\author{
A. H. DELSEMME \\ DEPARTMENT OF PHYSICS \& ASTRONOMY \\ THE UNIVERSITY OF TOLEDO \\ TOLEDO, OH 43606, U.S.A.
}

The origin of carbon and water on the terrestrial planets is not trivial, because their presence seems to be excluded by the high temperatures requested by the accretion disk models (Morfill 1988) as well as by Lewis' (1973) adiabat that can explain the different densities of the planets. These high temperatures imply that all dust was outgassed and dehydrated and most carbon stored in $\mathrm{CO}$, that is in the gas phase, before the separation of dust from gas, that occurred by dust sedimentation to the mid-plane of the accretion disk. Thermochemical equilibrium was easily reached, since chemical kinetics (Lewis \& Prinn 1980) had time constants shorter than the time needed to agglomerate the first planetesimals (Weidenschilling 1988).

Lewis et al. (1979) do not succeed in explaining the large retention of carbon by the Earth, even if they choose an adiabat in the accretion disk that brings the Earth's zone near the peak of graphite activity. But this peak is needed, not at one astronomical unit (AU) but near or beyond 2.6 AU, in order to explain the rich carbon chemistry of the carbonaceous chondrites -at least partially- by Fischer-Tropsch-Type reactions (Anders 1986). This alone strongly implies that the bulk of the observed carbon on the terrestrial planets has an exogenous origin and was brought about after the bulk of planetary accretion had ended.

The paradigm that has emerged to describe the origin of the Solar System implies that, during the final stages of planetary accumulation, the orbital evolution of the planetesimals, which is inevitable, automatically provides this source of carbon and water in the form of objects made at cooler temperatures because further away in the Solar System. At steady state, the mean relative velocities in the swarm of smaller bodies grow in proportion to the escape velocity of the largest body (Safronov 1969) inducing larger and larger orbital changes that widen the zone swept by the minor bodies. Because of the growth of Jupiter's embryo, the proto-Earth will be hit first by chondrites coming from the asteroid belt, then by comets coming from Jupiter's zone and eventually from Saturn's zone.

A simple model predicts on the Earth a veneer of $3-4 \mathrm{~km}$ of chondritic silicates, some $5 \mathrm{~km}$ of water, $1.2 \mathrm{~km}$ of organic compounds and an atmosphere of 240 bars. Comets bring $75 \%$ of the silicates, whereas they bring $98 \%$ of water and organic compounds and more than 99\% of the atmosphere. A major erosion of this veneer is to be expected from late giant impacts predicted by the accumulation models and also used to explain the formation of the Moon.

Some cosmochemical models of the terrestrial planets have independently converged to an inhomogeneous accretion, for instance the "chondrite model" (Larimer and Anders 1970). A recent version (Anders and Owen 1977) concludes that volatile elements were added at least on the Earth and Mars. Prinn and Fegley (1989) and Dreibus \& Wanke (1989) also recognize the need of an inhomogeneous accretion of two components whose origin they do not try to explain.

Since observational evidence has recently established the ubiquity of accretion disks around very young stars, and since we have just shown that there seems to be a general mechanism in accretion disks to make terrestrial planets and to bring them a veneer of water and organic compounds, it is tempting to conclude that the number of terrestrial planets ready to develop life-as-we-know-it, from water and carbon compounds, is extremely large in the Universe. 
Figure 1. Mid.plane temperature of the accretion disk as a function of radius. The crosses are Lewis 1974 aggregation temperatures of planets and satellites. The solid line is the adjustment of the disk model (Morfill 1988). The two dotted lines correspond to accretion rates 10 times smaller or larger.
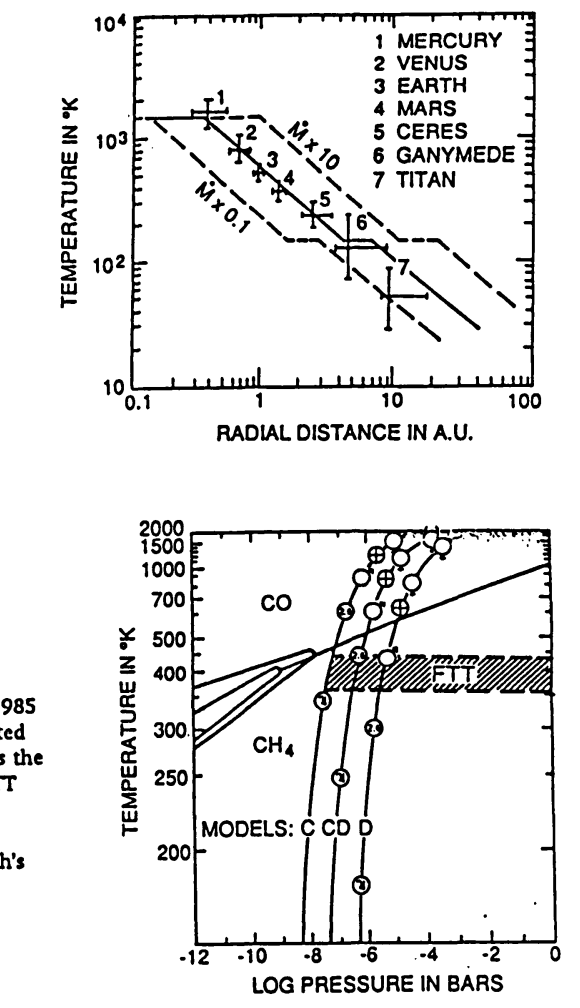

Figure 2. - Thermochemical equilibrium for carbon in a gas of solar composition. The quasi vertical curves are Cameron's 1985 adiabats. These adiabats can be interpreted as a cooling sequence. Adiabat $\mathrm{CD}$ brings the distance $2.6 \mathrm{AU}$ at the high end of the FTT temperature zone, implying that dust sedimentation took place at that time. The same adiabat shows that, in the Earth's zone (symbol $\oplus$ ) all carbon was then in gaseous CO.

\section{REFERENCES}

Anders, E. 1986, p. 31.39 in Comets Nucleus Sample Return, ESA SP 249.

Anders, E. and Grevesse, N. 1989 Geochim. Cosmochim. Acta, 53, 197-214.

Cameron, A.G.W. 1985, p. $1073-1099$ in Protostars and Planets II, Black and Matthews (eds), Univ. of Arizona Press, Tucson.

Chyba, C. F. 1987, Nature 330, 632-635.

Delsemme, A. H. 1981, p. 141-159 in Comets and the Origins of Life. Ponnamperuma (ed.), Reidel Publ., Dordreche.

Delscmme, A. H. 1984, Origins of Life 14, 51-60.

Delsemme, A. H. 1990a, in Comets in the Post-Halley Era, Newburn et al, (eds.) Kluwer Acad. Press, Dordrecht (in press).

Delsemme, A. H. 1990b, in Proc. COSPAR XXVIII Plenary Meeting. The Hague (in press).

Hayashi, C., Nakazawa, K., and Nakagawa, Y. 1985, p. 1100-1153 in Protostars and Planets II, Black and Matthews (cds.), Univ. of Arizona Press, Tucson.

Ip. W. H., and Fernandez, J. A. 1988, Icanus 74, 47-62.

Lewis, J. S. 1974, Science $186,440-443$.

Lewis, J. S., Barshay, S. S., Noyes, B. 1979, Icanus 37, 190-206.

Lewis, J. S. and Prinn, R. B. 1980, Astrophys. J. 238, 357-364.

Lin, D.N.C.. Papaloisou, J. 1985, p. $981-1072$ in Prolostars and Plancts II. Black and Matthews (eds.). Univ. of Arizona Press, Tucson.

Lynden-Bell, D., and Pringle, J. E. 1974, M.N. Roy. Astron. Soc. London 168, 603.637.

Matsui, T., and Abe, Y. 1986, Nature 322, 526-528.

Morfili, G. E. 1988, Icarus $75,371-379$

Morfill, G. E., Tschamuter, W., Volk, H. J. 1985, p. 493-533 in Protostars and Planets II, Black and Matthews (eds.). Univ. of Arizona Press, Tucson.

Morfill, G. E., and Wood, J. A. 1989, Icarus 82, 225-243.

Smith, B. A. and Terrilc, R. J. 1984, Science 226, 1421-1424.

Weidenschilling, S. J. 1988, p. 348-371 in "Metcorites and the Early Solar System", J. F. Kerridge and M. S. Matuhews, eds., Univ, of Arizona Press, Tucson.

Wood, J. A., and Morfill, G. E: 1988, p. 329-347 in "Metcorites and the Early Solar System", J. F. Kerridge and M. S. Mauhews, eds., Univ. of Arizona Press, Tucson. 